\title{
Studien über die Beziehung zwischen der Virulenz und den giftigen Leibessubstanzen der Mäusetyphusbazillen.
}

\author{
I. Mitteilung. \\ Von \\ Kôiti Yamamoto \\ (山本 耕 $\rightarrow$ ) \\ (Aus dem Bakteriologischen Institut der Kaiserlichen \\ Tohoku Universität zu Sendai, Direktor: \\ Prof. Dr. M. Kuroya.)
}

Seit längerer Zeit ist schon bekannt, dass ein Bakterium ein und derselben Art je nach dem Stamm nicht nur eine auffallende Virulenzschwankung zeigt, sondern manchmal auch hoch virulent werden kann, wenn es ein oder mehrere Tiere passiert hat. Wovon rührt denn solche Virulenzverschiedenheit her? Was man als die wichtigen Faktoren des Bestehens der "Virulenz" aufgezählt hat, ist die Fähigkeit des Mikroorganismus, erstens sich im lebenden Organismus zu vermehren und zweitens in ihm Gift $z u$ bilden. Letzteres führt nicht nur zur Intoxikation des angegriffenen Körpers, sondern liefert auch den Mikroorganismen eine bessere Bedingung zu ihrer Vermehrung im Körper, also sind die obenerwähnten Faktoren nicht voneinander unabhängig.

Als einer der Vorläufer auf diesem Gebiet veröffentlichte schon Kruse $\mathrm{e}^{1)}$, dass die durch Bakterien erzeugten "Angriffstoffe" die Abwehrstoffe des eingedrungenen Körpers neutralisieren und den Abwehrmechanismus des Lebewesens vernichten soll. Nach Wyssokowitsch hetzt das unter dem Einfluss von Bakterien erzeugte Ptomain die Zellenenergie des Körpers so weit herab, dass die Bakterien, die sonst nicht virulent waren, zu einer lebhaften Vermehrung veranlasst werden, was schliesslich den Tod der Tiere herbeiführt.

G a ma le i $a^{3)}$ fand dann auch, dass die Kaninchen, die sich bis dahin refräktär verhielten, durch gleichzeitige Verabreichung von Darmcholera und Prodigiosusextrakt oder Chemikalien, wie Nitrite, Nitrate, Leucin, angesteckt werden konnten. Von allen Theorien über diesen Gegenstand war seinerzeit die sog. „Aggressintheorie" am überzengendsten. Als erste beschäftigten sich $\mathrm{Kr}$ use 
und B on aduce $\mathrm{e}^{4)}$ mit diesen Substanzen und ermittelten, dass die Injektion abgetöteter Bakterien die Milzbrandinfektion erheblich beschleunige. Diese infektionbegünstigenden Stoffe nannte man "Lysine" und später "Aggressine." Andere Autoren, besonders $\mathrm{Bail}^{5}$ dehnten ihre Forschungen über die „Aggressine" noch ferner auf verschiedene Bakterien aus, auf sterilisierte Extrakte von Bakterien und wiesen die $\mathrm{Kr}$ u s e schen Substanzen nach. Es sei hier noch die Theorie von F. Gál $l^{\text {E) }}$ erwähnt, nach welcher die Vergiftung durch die Spaltungsprodukte des Bakterieneiweisses, Peptone und Endprodukte, die unter Einwirkung von Fermenten im Organismus entstehen, zustande kommt. Was die Virulenzverschiedenheit betrifft, so erklärte er, dass virulente Bakterien im infizierten Organismus einen stärkeren Reiz zur Fermentproduktion ausüben, als nicht virulente, so dass aus virulenten Bakterien mehr Spaltungsprodukte sich bilden, als aus nicht virulenten. Er konnte nachweisen, dass Fermente, wie Trypsin und Erepsin die Virulenz des Collibacillus erhöhen. Thiel und Embel to $n^{7}$ erklarten, dass das Bakterienprotoplasma infolge von proteolytischer Degeneration eine toxische Substanz liefert und, dass die echte Virulenz von dem Vermögen abhängt, eine Kapsel zu bilden.

Was als ein Faktor der Bakterienvermehrung im Körper noch in Betracht kommt, ist die Adaptation. Wenn die Lebensbedingungen dem Erreger ungüstig sind, so bekommt er die Fähigkeit, sich der Umwelt anzupassen und dort zu vermehren. Solche Bakterien, also serumfeste Bakterien sind zuerst ron Jensen ${ }^{8}$, Wassermann und Ostertag $g^{9}$, und von Jaffé und Bessere ${ }^{10)}$ bei verschiedenen Bakterienarten beobachtet worden.

Manche von den oben erwähnten Autoren haben versucht, die Virulenz mit der Kapselbildung in Zusammenhang zu bringen, und so sahen Preisz ${ }^{11}$, Gruber und Futaki ${ }^{21}$, und $\mathrm{Bail}^{13\}}$, dass die abgeschwächten Varietäten der Milzbrandbazillen gar keine oder fast keine Kapsel bildeten. Stamatin ${ }^{41}$, $\mathrm{Schaef} \mathrm{r}^{15)}$, Sterne $\mathrm{e}^{16)}$, von $\mathrm{Buz} \mathrm{a}^{17)}$, und andere Autoren stellten ferner fest, dass sich die starkvirulenten Milzbrandbazillen im empfänglichen Tiere mit einer Kapsel umgeben, besonders der letztgenannte von ihnen sagt in seiner neulich erschienenen Mitteilung, dass die Virulenz des Anthraxbazillus nicht nur durch die Kapselbildung, sondern auch durch haemopeptische Eigenschaft bedingt ist.

Der Verfasser lenkte daher sein Augenmerk auf die Zusammensetzung der Körpersubstanzen der zwei verschieden stark virulenten Mäusetyphusstämme und versuchte auf diese Weise, den Schlüssel zur Lösung des Virulenzproblems zu finden.

Von den Autoren, wie Voges ${ }^{18)}$ und $\mathrm{K}$ osk $\mathrm{e}^{(9)}$, wurde schon erkannt, dass in alter Bouillonkultur der Paratyphus-Enteritidis-Gruppe die toxische Substanz enthalten ist. Branham ${ }^{20)}$ veröffentlichte auch, dass der flüssige Nährboden von $B$. enteritidis und ihm nahe verwandten Bakterien auf Kaninchen und Mäuse toxisch einwirkt, und die daraus isolierte proteinfreie Substanz erst durch das Zerfallen der Bakterienleiber frei gemacht wird. Ec k e $\mathrm{r}^{22}$ berichtete schon im Jahre 1917 über die toxische Eigenschaft der 24stündigen Kultur der Paratyphusbazillen. Die intrakutane Verimpfung von Extrakten, die Ecker 
und Rimington ${ }^{22)}$ aus Breslaubazillen gewannen, mit wenig Stickstoff und viel Kohlenhydraten, riefen bei Kaninchen die Hautreaktion hervor.

Die von Casper ${ }^{23)}$ mit Antiformin gewonnenen Breslaubazillenextrakte waren sehr toxisch für Mäuse bei intraperitonealer Injektion. Ca s per schrieb die toxischen Eigenschaften dieser Extrakte ihrer polysaccharidartigen CSubstanz zu.

Menten und $\mathrm{Kin} \mathrm{g}^{24)}$ gewannen aus Berkefeld-Filtraten von Paratyphus B-Bazillen mittels konzentrierter Essigsäure eine phosphor- und schwefelfreie, toxische Fraktion.

Furth und Landstein e $\mathrm{r}^{25)}$ berichteten, dass unter den aus Paratyphusarten isolierten Substanzen mit verschiedenen chemischen und serologischen Eigenschaften nur die mit dem Proteincharakter toxisch war.

$\mathrm{Ra}$ istrick und Tople $\mathrm{y}^{26}$ befassten sich sehr eingehend mit diesen Forschungen. Sie gewannen aus dem abgetöteten, azetonextrahierten und getrockneten Bazillenpuiver von Bact. aertrycke verschiedene Fraktionen, unter denen die Fraktion mit 68 Proz. Alkohol behandelte Fraktion (Zeichen:68) einen guten Immunisierungseffekt zeigte. Nach chemischer Analyse zeigte bei immunologisch wirksamen Fraktionen der Zuckergehalt 28,8-37,8 Proz. Die Präparate können kaum intaktes Protein enthalten, da die Eiweissreaktion, abgesehen von Ninhydrin- und Biuretprobe, negativ ausfiel. Ob die toxisch und immunologisch wirksame Substanz ein und dieselbe ist, oder ob es sich um mehrere Substanzen handelt, bleibt nach ihnen noch ungeklärt.

Boivin und Mesrobean $\mathbf{n}^{27}$ berichteten im Jahre 1935 über die Ergebnisse von Untersuchungen, in deren Verlauf es ihnen gelungen ist, aus den mit Trichloressigsäure und nachfolgendem Azetonzusatz behandelten AertryckeKulturen eine für Mäuse stark toxisch wirkende und das antibakterielle Serum bis zu hoher Verdünnung präzipitierende Substanz herauszugewinnen. Diese Substanz, die sie als "Antigène complet" bezeichneten, zerlegte sich beim Kochen in schwach saurer Reaktion in freie Fettsäure und in Polysaccharid. Dieses Polysaccharid präzipitiert hoch spezifisch entsprechendes antibakterielles Serum, jedoch ist es deshalb nicht immunogen im echten Sinne des Wortes, weil es im Tierkörper keine Antikörper auslösen kam. Ivánovics ${ }^{28}$ erhielt aus Agarkulturen der Breslaubazillen mittels kalter Essigsãure sehr toxische Extrakte, die nach Hydrolyse durchschnittlich eine 20 Proz. frei werdende reduzierende Substanz lieferten. Ihr Stickstoffgehalt betrug ca. 3 Proz. Bei ihnen erwies sich von Eiweissreaktionen nur die Biuretprobe als positiv. Auch bei uns kamen Hosoya und $\mathrm{Kaw}$ a sima $a^{(9)}$ infolge der Untersuchungen über die giftigen Substanzen der Typhus, Paratyphus A- und BBazillen zu fast gleichen Resultaten mit den oben genannten Autoren.

\section{Versuche.}

Die zu den Versuchen verwendeten Stämme.

$Z u$ diesen Untersuchungen wurden zwei seit Jahren in der Sammlung unseres Instituts befindliche Mäusetyphusstämme, deren einer 
als „Ms $166^{\prime \prime}$, der andere als „Ms 163 “ bezeichnet ist, herangezogen. Die Virulenz des ,Ms $166^{“}$ war bezogen auf Mäuse niederwertig, mit dessen $0,1 \mathrm{mg}$ geimpft, erst die Tiere alle zugrunde gingen, während die des „Ms 163“, dessen M.L.D. anfänglich 0,02 mg betrug, aber durch 20 mal wiederholte Tierpassage zu $0,0005 \mathrm{mg}$ gesteigert wurde, sehr

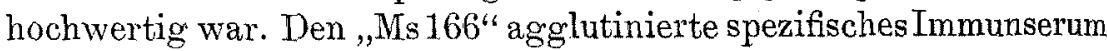
bis 1:20000, dagegen den ,Ms 163" bis 1:5000. Beide Stämme zeigten auf Endoschem Agar typische S-förmige Kolonien. Die Bazillen wurden auf gewöhnlichen Agarnährböden von $\mathrm{pH} 7,2$, die mit Rinderfleisch hergestellt wurden, bei $37^{\circ} 24$ Stunden lang gezüchtet und für jeden Versuch wurden $161 \mathrm{~g}$ von, ,Ms 166 " und $208 \mathrm{~g}$ von „Ms 163 " gebraucht.

\section{Methodikzur Herstellungdes Bazillenextraktes.}

In diesem Versuche wurde zur Herstellung des Bazillenextraktes ein eigenartiges Verfahren nach $\mathrm{K}$ u ro $\mathrm{ya}^{30}{ }^{30}$, welches aus dem Waschen und Umrühren, und dem darauf folgenden Zerreiben der Bakterienmasse besteht, vorgenommen.

Zuerst hatte man eine kleinere Portion der Kulturen bei $80^{\circ}$ in Luftbad zu konstantem Gewicht getrocknet und dadurch das Trockengewicht der Mengen der ganzen Kulturen ausgerechnet. Dann wurde die Bakterienmasse in einem grossen Becherglas mit 1,5 l destilliertem Wasser aufgeschwemmt und die so erhaltene Suspension etwa 5-6 Stunden lang tüchtig umgerührt. Es soll auf diese Weise der die Oberfläche des Bakterienleibes bildende Teil in Wasser losgelöst werden. Die abgeschleuderte Bakterienmasse wurde nochmals in Wasser homogen suspensiert und abermals etwa 15 Minuten lang zentrifugiert. Diese Manipulation wiederholte sich so oft, bis die letzte klare obere Flüssigkeit auf Zusatz von Trichloressigsäure keine Trübung mehr zeigte. Die angesammelte Flüssigkeit, die die Bakterien noch in beträchtlicher Menge enthielt, wurde dann mit Hilfe von, Sharpless"Zentrifuge von 14000 Umdrehungen pro Minute abgeschleudert. Die so extrahierte Flüssigkeit war bei $\mathrm{pH}$ 6,6-7,0 und stellte einen gelblichen Farbenton dar. Je $10 \mathrm{ccm}$ dieses Extraktes wurde dann in 3 PetriSchalen abgetrocknet und aus dem durchschnittlichen Wert die gesamte Menge der ins Wasser ausgezogenen Substanzen ungefähr ausgerechnet. Die so erhaltene Lösung wurde zum Schluss mit Toluol vermischt und dann in dunkler Eiskammer stehengelassen, um die in der Lösung noch lebend enthaltenen Bakterien abzutöten. Die so her- 
gestellte Lösung nennt man provisorisch „Extrakt I".

Der abgeschleuderte Bakterienrest schliesst in sich noch eine Menge zu extrahierende Substanzen, die erst nach dem Zerfall des Leibes in Freiheit gesetzt werden können. Zu diesem $Z$ wecke wurde die Bakterienmasse im Exsikkator über $\mathrm{CaCl}_{2}$ mässig entwässert, im Mörser mit gleicher Menge von Quarzsand so lange (ungefähr 5-6:Stunden) tüchtig. zerrieben, bis die Bakterienmasse zäh und sch wer mazerierbar geworden war, wobei sie sich unter dem Mikroskop als nur homogen gefärbtes Protoplasma darstellte. Die zerriebene Bakterienmasse wurde dann mit 1 1 destilliertem Wasser aufgeschwemmt und nach ganz gleicher Manipulation wie beim Extrakt I behandelt, was dann „Extrakt II" ergab.

\section{Giftigkeit der aus beiden Stämmengew on- nenen Extraktefür Mäuse.}

Je 1,0 ccm, 0,5 ccm, 0,2 ccm, 0,1 ccm, .... dieser Extrakte, dessen einer Waschwasserextrakt der Bakterienleiber, der andere ein Extrakt der mazerierten Bakterienreste war, erhielten Mäuse intraperitoneal eingespritzt. Wie aus Tabelle 1 ersichtlich ist, betrug M.L.D. des Extraktes I und II aus Stamm „Ms 166“ in Trockengewicht gerechnet, $0,24 \mathrm{mg}$ u. 0,5 mg, dagegen die aus Stamm ,Ms 163 “ $0,75 \mathrm{mg} \mathrm{u.} 0,31 \mathrm{mg}$. Es besteht also fast kein deutlich Unterschied zwischen beiden Extrakten in bezug auf ihre Giftigkeit.

Nach der Injektion lagen die Tiere mit geschlossenen Augen und beschleunigter Atmung unruhig da und gingen unter allmählicher Schwäche in 5-38 Stunden ein.

Hier ist höchstens beachtenswert, dass sich aber bei zwei Mäusen, die Extrakt II aus Stamm "Ms 163" bekamen, akute typische Nervensymptome einstellten. Etwa 1 Stunde nach der Injektion gerieten die Tiere plötzlich in einen Erre-

Tabelle 1 .

Giftigkeit der aus 2 verschiedenen Stämmen extrahierten Extrakte für Mäuse.

\begin{tabular}{|c|c|c|c|c|c|}
\hline \multirow{2}{*}{\multicolumn{2}{|c|}{$\begin{array}{l}\text { Stämme } \\
\text { Extrakt }\end{array}$}} & \multicolumn{2}{|c|}{ "Ms $166^{\prime 6}$} & \multicolumn{2}{|c|}{ "Ms $163^{k}$} \\
\hline & & I & II & $\mathrm{I}$ & II \\
\hline \multicolumn{2}{|c|}{ TG per $1 \mathrm{ccm}$} & $1,2 \mathrm{mg}$ & $2,5 \mathrm{mg}$ & $1,5 \mathrm{mg}$ & $3,13 \mathrm{mg}$ \\
\hline \multirow{5}{*}{ 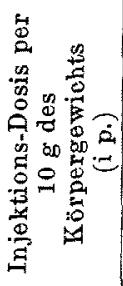 } & $1,0 \mathrm{ccm}$ & $1 / 1$ & $1 / 1$ & $3 / 3$ & $1 / 1$ \\
\hline & 0,5 & $3 / 3$ & $2 / 2$ & $3 / 3$ & $2 / 2$ \\
\hline & 0,2 & $3 / 3$ & $3 / 3$ & $2 / 3$ & $3 / 3$ \\
\hline & 0,1 & $2 / 3$ & $1 / 3$ & $1 / 3$ & $3 / 3$ \\
\hline & 0,05 & $1 / 3$ & & & $2 / 3$ \\
\hline \multicolumn{2}{|c|}{ M. L. D. } & $0,24 \mathrm{mg}$ & $0,5 \mathrm{mg}$ & $0,75 \mathrm{mg}$ & $0,31 \mathrm{mg}$ \\
\hline
\end{tabular}

$3 / 3$ bedeutet, dass unter 3 gebrauchten Tieren allo 3 gestorben sind, $\mathfrak{u}$. desgl. 
gungszustand, liefen hier und da aufspringend fort und endeten schliesslich unter Krampf und Paräse der hinteren Extremitäten letal. Bei der Sektion sah man in fast allen Fällen die Hyperämie des Peritoneums und der Dünndärme, besonders zeichnete sich die Milz durch ihren rötlichvioletten oder noch häufiger dunkelvioletten Ton aus. Da

Tabelle 2.-3.

Intrakutane Reaktion durch Extrakte aus Stamm ,Ms 166".
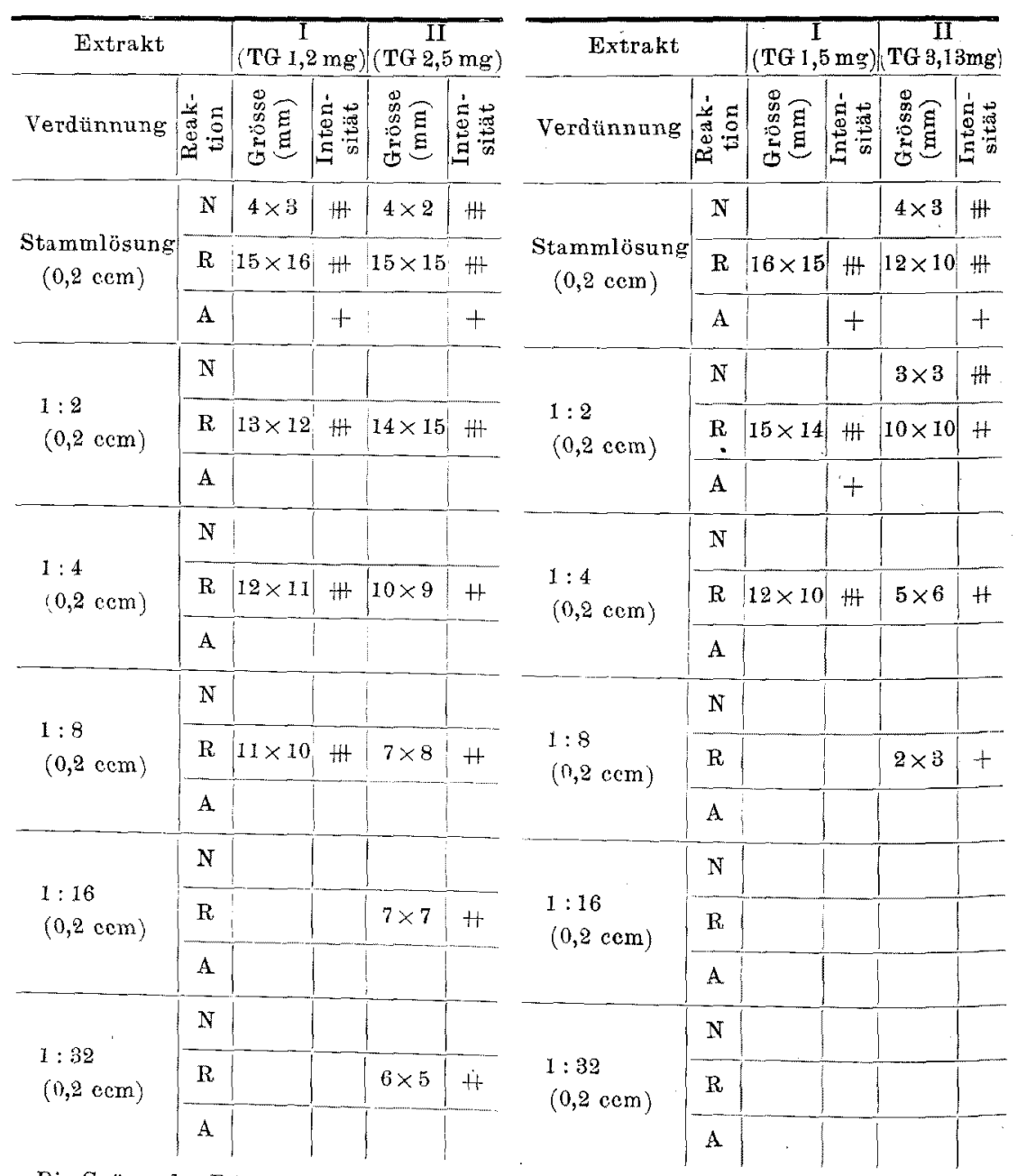

Die Grösse der Rötung und der Nekrose sind in mm ausgedrückt. $\mathrm{N}=$ Nekrose 
aber ihr Gewicht, abgesehen von einem Fall, leichter als 0,1 g war, wies sie keine so deutliche Anschwellung auf, vorausgesetzt, dass das Gewicht der Milz von normalen Mäusen im Durchschnitt $0,1 \mathrm{~g}$ wiegt. Diarrhoe wurde bei Extrakten aus Stamm ,Ms $166^{\prime \prime}$ bei 5 unter 17 Todesfällen, hingegen bei den aus Stamm „Ms $163^{\prime \prime}$ bei 8 unter 20 Todesfällen beobachtet. Die Aszitesansammlung liess sich in gar keinem Fall erkennen. Die Kultur aus Herz- und Milzblut fiel immer negativ aus.

\section{Intrakutane Reaktionsfähigkeit des Extraktes} I und II aus beiden Stämmen.

Jeder Extrakt wurde nach Zusatz von $\mathrm{NaCl}$ in Konzentration von 0,85 Proz. mit physiologischer Kochsalzlösung verschieden verdünnt, wie es in der Tabelle 2 und 3 dargestellt ist, in einer Dosis von $0,2 \mathrm{ccm}$ in den Rücken der gesunden Meerschweinchen intrakutan injiziert und das Resultat nach 48 Sturiden beobachtet.

Die Rötungen fand man bei Extrakten aus Stamm ,Ms 166" noch in Verdünnung 1:8-1:32, dagegen bei Extrakten aus Stamm ,"Ms 163" nur in Verdünnung $1: 4-1: 8$. Die grösste Rötung zeigte $15 \times 16 \mathrm{~mm}$. Ausserdem fand man manchmal Nekrose und Anschwellung, besonders in höherer Konzentration. Im allgemeinen bemerkt man, dass in bezug auf die Hautreaktion beide Extrakte II stärker wirksam sind als Extrakte I und insbesondere Extrakt II aus „Ms 166" am stärksten aktiv ist. Diese Tatsache, wie nachher wieder erwähnt, zeigt, dass die Hautreaktionsfähigkeit dem PF-Gehalt in der Lösung zuzuschreiben ist.

\section{Isolierung dex in Extrakten enthaltenen Fraktionen.}

Wird ein Bazillenextrakt mit Säure versetzt, so tritt im allgemeinen eine homogene Trübung, ja sogar ein flockiger Niederschlag in einer geeigneten $\mathrm{pH}-\mathrm{Z}$ one auf, welcher im isoelektrischen Punkte bis zum Maximum gelangt. Die Bestimmung des Trübungsgrades geschah in der Weise, dass man in eine Reihe von Reagenzgläsern je $0,9 \mathrm{ccm}$ des Extraktes verteilte, durch Zusatz von Essigsäure von verschiedener Konzentration innerhalb $0,1 \mathrm{ccm}$ verschieden abgestufte $\mathrm{pH}$ gab und sie zuletzt mit destilliertem Wasser so ergänzte, dass die Gesamtmenge gerade $10 \mathrm{ccm}$ erreichte und jede entstehende Trübung mittels Nephelometer de A. Boudouin et H. Bénard gemessen wurde (Fig. 1). Auf diese Weise erfuhr man, dass der isoelektrische Punkt für beide Ex- 
trakte, I und II aus Stamm „Ms 166" bei $\mathrm{pH} 3,7$ - und $\mathrm{pH} 4,4$ liegt. Über die beiden Extrakte aus Stamm ,Ms 163" wird es später beschrieben.

\section{(A) Isolierung der Fraktionen der Extrakte I und II aus Stamm ,Ms 166".}

Nach Figur 1 wurden beide Extrakte aus „Ms 166“ mit Essigsäure zu pH 3,7 oder 4,4 gebracht und 5 Stunden lang in Zimmertemperatur stehen gelassen. Der abgeschleuderte Niederschlag wurde dann unter allmählichem Zusatz von $\mathrm{N} / 5$ $\mathrm{NaOH}$ und wenig Wasser gelöst, dann wieder mit Essigsäure in geeigneter $\mathrm{pH}$ gefällt. Diese Manipulation wiederholte sich so lange, bis die letzte überstehende Flüssigkeitkeine Molis ch sche Reaktion mehr zeigte. Bei dieser Reinigung fand eine Verschiebung der $\mathrm{pH}$ (anfänglich 3,7

Fig. 1.

Trübungsgrad der Extrakte aus sehwach virulenten Stamm ,Ms $166^{\prime \prime}$ (mit $\mathrm{CH}_{5} \mathrm{CO}_{2} \mathrm{H}$ versetzt).

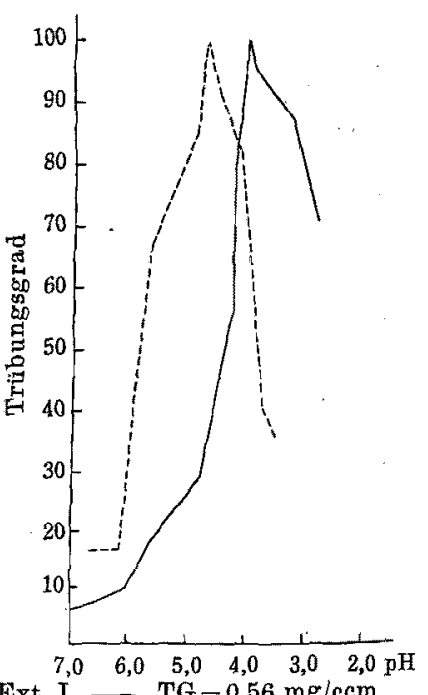

Ext. I $-\mathrm{TG}=0.56 \mathrm{mg} / \mathrm{ccm}$ Ext. II … TG $=1.5 \mathrm{mg} / \mathrm{ccm}$ oder 4,4 , später 3,9 oder 5,0 ) statt. Das in solcher Weise gereinigte Eiweiss wurde dann erneut gelöst, mit Toluol übergeschichtet und in dunkler Eiskammer aufbewahrt. Diese Fraktion bezeichnete man vorläufig als , $\mathrm{PF}_{1}$ “. Jede Flüssigkeit, die durch Ansäuerung und Zentrifugieren vom Niederschlag ausgeschieden worden war, wurde nun angesammelt, neutralisiert und unter vermindertem Druck bei niedriger Temperatur $\left(37^{\circ}-40^{\circ}\right)$ auf $1 / 5$ ihres Anfangsvolumens konzentriert, dann wieder mit Süure zu pH 3,7 oder 4,4 gebracht und abgeschleudert. Der so gewonnene Niederschlag wurde zu PF $\mathrm{PF}_{\mathbf{1}}$ hinzugesetzt.

Da hierbei die abgetrennte überstehende Flüssigkeit noch getrübt war, wurde diese nun weiter mit 50 Prozent. Trichloressigsäure $(\mathrm{pH} 1,2)$ angesäuert, dann stark abzentrifugiert und dadurch die klare überstehende Flüssigkeit und der Niederschlag voneinander vollkommen abgetrennt.

Dieser Niederschlag zerfiel ferner in zwei Fraktionen, von denen die eine mit Bezeichnung „, $\mathrm{PF}_{2}$ " wieder mit Essigsäure bei $\mathrm{pH} 3,7$ oder. 4,4 , und die andere dagegen mit Bezeichnung „,PF ${ }_{3}$ " nur mit Trich- 
Fig. 2.

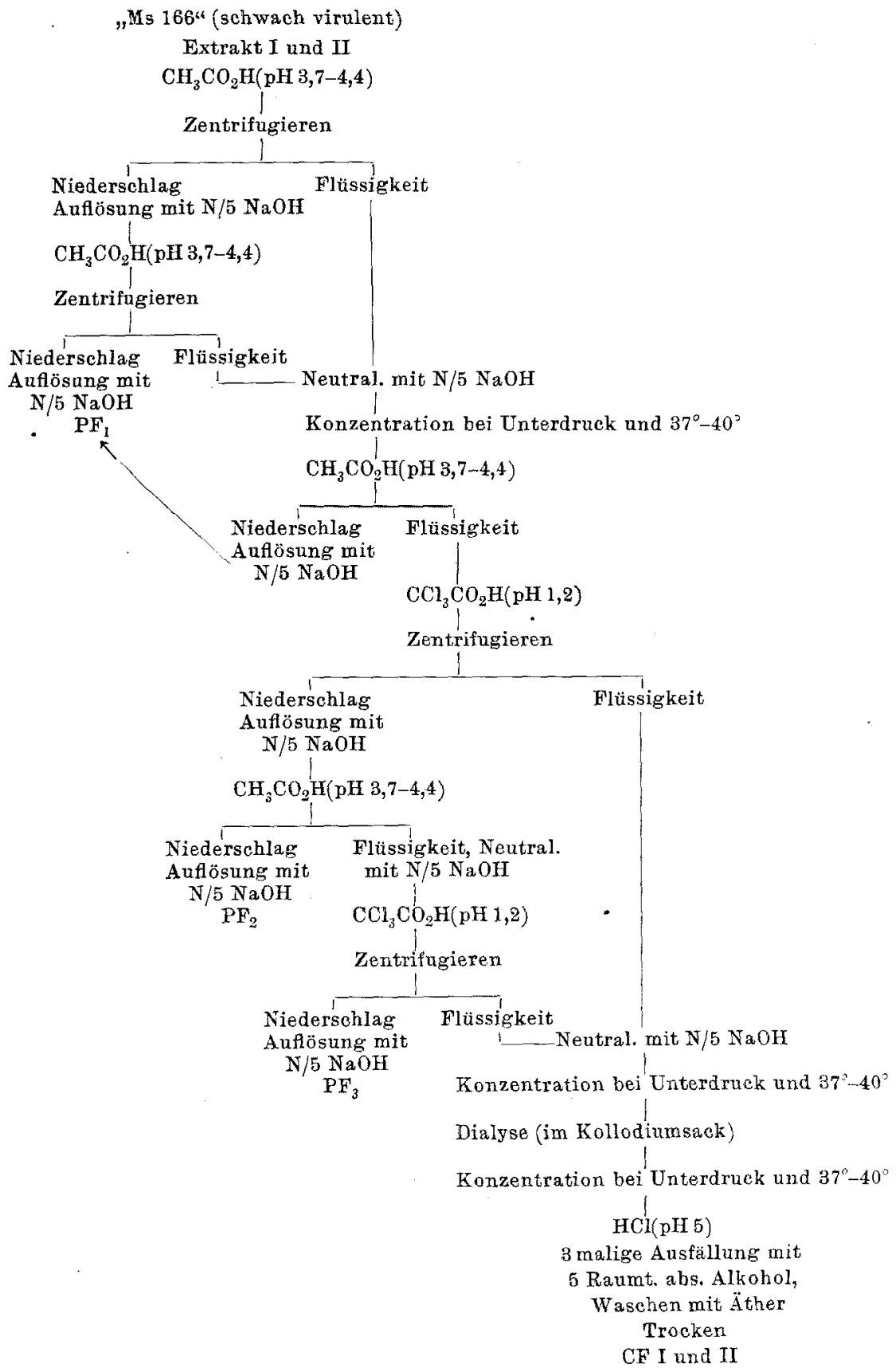


loressigsäure $\left(\mathrm{pH} \mathrm{1,2)} \mathrm{gefällt} \mathrm{wurde.} \mathrm{Die} \mathrm{PF}_{2}\right.$ und $\mathrm{PF}_{3}$ wurden nach wiederholter Reinigung ebenso wie $\mathrm{PF}_{1}$ aufbewahrt.

Nach der Entfernung der auf diese Weise vollkommen ausgezogenen PF-Fraktionen, wurde die Lösung wieder angesammelt und neutralisiert. Diese wurde wiederum bei Unterdruck und $37^{\circ}-40^{\circ}$ eingeengt und in einem Kollodiumsack 4 Tage lang gegen Leitungswasser dialysiert. Die so behandelte Lösung wurde-wie dies im folgenden bei jeder alkoholischen Fällung üblich war-mit Salzsäure bis pH 5,0 angesäuert und mit 5 Raumteilen abs. Alkohol zur Fällung gebracht.

DerNiederschlag wurde erneut wiederum in wenig Wasser gelöst. Diese Fällung wurde 3mal wiederholt und zuletzt nach dem Waschen mit Ather im Exsikkator vollständig getrocknet. Die so gewonnene Substanz nennt man „CF I und II" (Über Obengeschildertes siehe Fig. 2).

(B) Isolierung der Fraktionen der Extrakte I und II aus Stamm ,Ms $163^{\prime \prime}$.

Die Extrakte aus diesem Stamm unterscheiden sich von den aus Stamm „Ms 166" dadurch, dass es durch Ansäuerung zu keiner Fällung kommt. Dies bedeutet, dass zwischen den extrahierten Substanzen aus beiden Stëmmen nicht nur quantitativ, sondern auch qualitativ eine grosse Differenz vorhanden sein muss, mit anderen Worten, ein stark virulenter Stamm vom schwach virulenten strukturell verschieden ist. Also ist das Isolierungsverfahren der Fraktionen vom obigen im wesentlichen abweichend.

a) Isolierung der Fraktionen aus Extrakt I.

Da bei diesem Extrakt nur durch Trichloressigsäure ( $\mathrm{pH}$ 1,2) eine kaum erkennbare Trübung auftrat, so ging die Behandlung folgendermassen vor sich. Die Lösung wurde unter vermindertem Druck und bei $37^{\circ} \mathrm{C}-40^{\circ} \mathrm{C}$ bis auf etwa $1 / 20$ des Anfangsvolumens eingeengt und deren kleinere Portion wieder zur Trübungsprobe herangezogen, wobei sich ergab, dass sowohl durch 10 Proz. Trichloressigsäure (pH 3,7) als

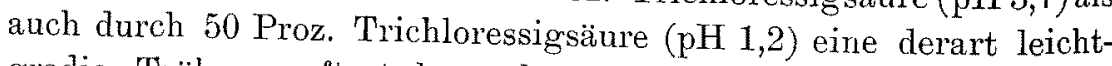
gradige Trübung auftrat, dass es kaum zur Bildung der Trübungskurve kam. Diese zur. Probe angestellte Lösung wurde wieder angesammelt, neutralisiert und in einer Kollodiummembran gegen Leitungswasser 4 Tage lang dialysiert, und dann nochmals bei vermindertem Druck konzentriert. Nach dem gleichen Verfahren mit Salzsäure, Alkohol 
und $\ddot{A}$ ther wie oben, wurde aus dieser Lösung eine weissliche Substanz "CF I" herausgewonnen.

b) Isolierung der Fraktionen aus Extrakt II.

Da eine leichte Trübung durch Salzsäure, Essigsäure und Trichloressigsäure auftrat, so wurde die Lösung bis auf etwa $1 / 14$ ihres Volumens konzentriert und dann erfolgte damit die Bestimmung der Trübungskurve mit Essigsäure (Fig. 3). Die Trübung erreichte dabei bei pH 4,4 den höchsten Wert, doch sie brachte keinerlei Niederschlag mit sich. Im Gegensatz dazu sah man durch Ansäuerung mit Trichloressigsäure $(\mathrm{pH}$ 1,2) eine kleine Menge Niederschlag entstehen. Dieser Niederschlag wurde mittels $\mathrm{N} / 5 \mathrm{NaOH}$ gelöst, die Lösung fällte nicht wiederum durch Essigsäure, sondern nur durch Trichloressigsäure $(\mathrm{pH} 1,2)$.

Fig. 3.

Tribungsgrad des eingeengten Extraktes II aus "Ms $163^{\prime \prime}$ (mit $\mathrm{CH}_{3} \mathrm{CO}_{2} \mathrm{H}$ versetzt).

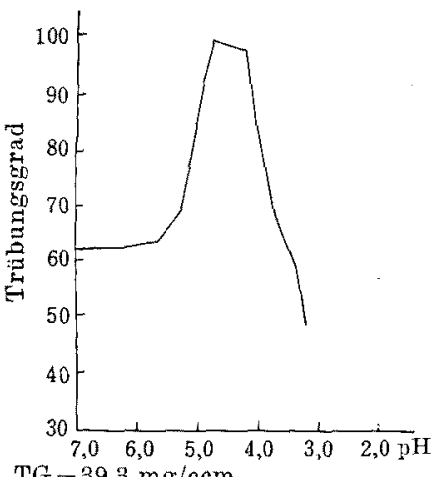
$\mathrm{TG}=39,3 \mathrm{mg} / \mathrm{ccm}$

Diese Substanz wurde mit Bezeichnung „PF“ in getrocknetern Zustand aufbewahrt. Während mehrmals wiederholter Reinigung

Fig. 4.

,Ms 163" (stark vìralent)

Extrakt I

Konzent. b. Unterd. u. $37^{\circ}-40^{\circ}$

auf ea $1 / 20$ des Volum.

Dialyse (im Kollodiumsack)

Konzent. b. Unterd. u. $37^{2}-40^{\circ}$ $\mathrm{HCl}(\mathrm{pH} 5)$

3 malige Ausfällung mit 5

Raumt. abs. Alkohol und

Waschen mit Äther

Trocken

$\mathrm{CF}$ I der PF wurde die überstehende Flüssigkeit, die anfänglich einen milchweisslichen Ton zeigte, helldurchsichtig. Diese Flüssigkeit wurde dann wieder angesammelt und bei vermindertem Druck konzentriert. Nachdem man dann diese Flüssigkeit nach der Dialyse mit Salzsäure, Alkohol und Äther behandelt hatte, wurde sie teils in Lösung, teils in Gestalt von Trockensubstanz gehalten. Diese Fraktion nennt man „CF II“. Die obengeschilderten Verfahren veranschaulichen Fig. 4 und 5 .

Giftigkeit der Fraktionen.

(A) Giftigkeit für Mäuse.

Jede Fraktion-gewonnen aus beiden Stämmen-wurde in gleicher Weise wie bei Extrakten den Mäusen intraperitoneal eingespritzt. 


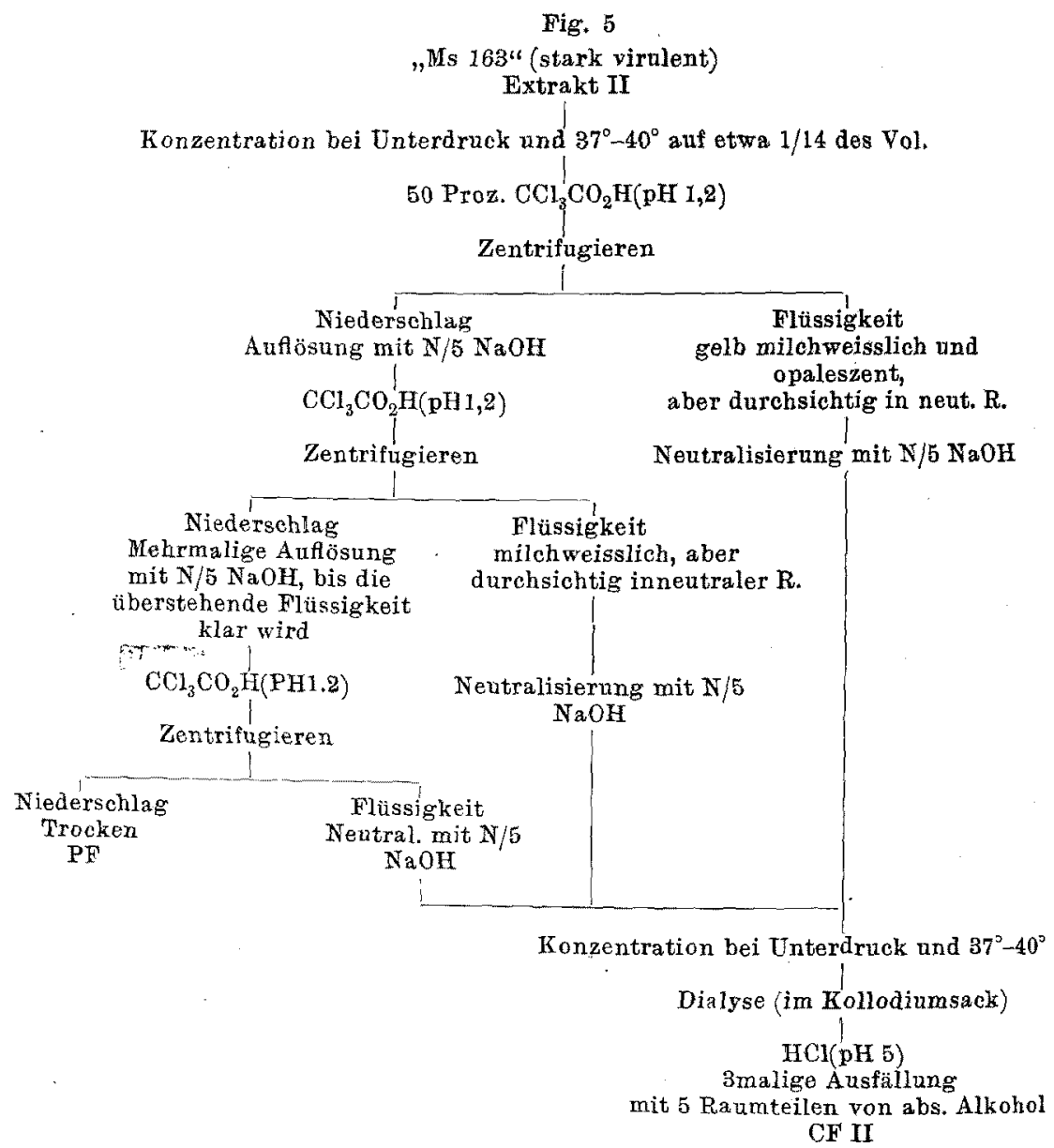

Wie aus Tabelle 4 und 5 ersichtlich ist, ist M. L.D. der CF I und II aus "Ms 166 " $0,2 \mathrm{mg}$ und $0,5 \mathrm{mg}$, während $\mathrm{PF}\left(\mathrm{PF}_{1}, \mathrm{PF}_{2}\right.$, und $\mathrm{PF}_{3}$ ) bei demselben Stamm $2 \mathrm{mg}-0,5 \mathrm{mg}$ betrug. Bei ,Ms 163" betrug M.L.D. von CF I und II, und PF 0,2 $\mathrm{mg}, 0,2 \mathrm{mg}$, und $2 \mathrm{mg}$.

Im allgemeinen also ist CF (M. L. D. 0,2 $\mathrm{mg}-0,5 \mathrm{mg}$ ) giftiger als PF (M.L.D. 0,5 mg-2 mg) und alle mit Trichloressigsäure isolierten PF sind schwächer giftig als die anderen.

Der Sektionsbefund ergab keinen deutlichen Unterschied im Vergleich mit dem bei den durch Extrakte eingegangenen Tieren, nur dass sich die Hyperämie der Nebenniere und die Diarrhoe bei den eine deutliche Eiweissreaktion zeigenden Fraktionen wie PF einstellten. Aber es ist doch noch fraglich, ob solch ein Befund für diese Fraktionen als 
Tabelle 4.

Giftigkeit der aus Extrakten von schwach virulentem Stamm "Ms $166^{\circ}$ isolierten Fraktionen für Mäuse.

\begin{tabular}{|c|c|c|c|c|c|c|c|c|c|}
\hline \multicolumn{2}{|c|}{ Extrakt } & \multicolumn{4}{|c|}{ I } & \multicolumn{4}{|c|}{ II } \\
\hline \multirow{7}{*}{ 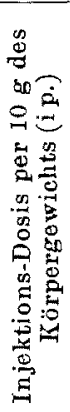 } & $\begin{array}{c}\text { Frak- } \\
\text { tion }\end{array}$ & CF & $\mathrm{PF}_{1}$ & $\mathrm{PF}_{2}$ & $\mathrm{PF}_{3}$ & CF & $P F_{1}$ & $\mathrm{PF}_{2}$ & $\mathrm{PF}_{3}$ \\
\hline & $4,0 \mathrm{mg}$ & & $1 / 1$ & $1 / 1$ & $1 / 1$ & & $1 / 1$ & $1 / 1$ & $1 / 1$ \\
\hline & 2,0 & & $2 / 2$ & $2 / 2$ & $3 / 3$ & & $3 / 3$ & $3 / 3$ & $3 / 3$ \\
\hline & 1,0 & $1 / 1$ & $3 / 3$ & $3 / 3$ & $1 / 3$ & $1 / 1$ & $3 / 3$ & $2 / 3$ & $2 / 3$ \\
\hline & 0,5 & $3 / 3$ & $2 / 3$ & $3 / 3$ & & $3 / 3$ & $2 / 3$ & $1 / 3$ & \\
\hline & 0,2 & $3 / 3$ & & $2 / 3$ & & $2 / 3$ & & & \\
\hline & 0,1 & $2 / 3$ & & & & & & & \\
\hline M. & & $0,2 \mathrm{mg}$ & $1,0 \mathrm{mg}$ & $0,5 \mathrm{mg}$ & $2,0 \mathrm{mg}$ & $0,5 \mathrm{mg}$ & $1,0 \mathrm{mg}$ & $2,0 \mathrm{mg}$ & $2,0 \mathrm{mg}$ \\
\hline
\end{tabular}

$3 / 3$ bedeutet, dass unter 3 gebrauchten Tieren alle 3 gestorben sind, u. desgl.

Tabelle 5 .

Giftigkeit der aus Extrakten von stark virulentem Stamm, "Ws $163^{\prime \prime}$

isolierten Fraktionen für Mäuse.

\begin{tabular}{c|c|c|c|c}
\hline \multicolumn{1}{c|}{ Extrakt } & I & \multicolumn{2}{|c}{ II } \\
\hline Fraktion & CF & CF & PF \\
\hline & $2,0 \mathrm{mg}$ & & & $3 / 3$ \\
\cline { 2 - 5 } & 1,0 & $3 / 3$ & $3 / 3$ & $1 / 3$ \\
$\begin{array}{c}\text { Injektions-Dosis } \\
\text { per 10 g des }\end{array}$ & 0,5 & $3 / 3$ & $3 / 3$ & $0 / 1$ \\
$\begin{array}{c}\text { Körpergewichts } \\
\text { (i p.) }\end{array}$ & 0,2 & $3 / 3$ & $3 / 3$ & \\
\cline { 2 - 5 } & 0,1 & $2 / 3$ & $1 / 3$ & \\
\hline M. L. D. & 0,05 & $2 / 3$ & & \\
\hline
\end{tabular}

$3 / 3$ bedeutet, dass unter 3 gebrauchten

Tieren alle 3 gestorben sind, u. desgl. charakteristisch gilt oder nicht.

(B) Intrakutane Reaktion.

Mit 0,85 Proz. Na Cl-Lösungen wurden sowohl CF I und II, und $\mathrm{PF}_{1} \mathrm{I}$ und II aus „Ms 166“, als auch CF I und II aus , Ms 163 " derart verdünnt, dass je $0,8 \mathrm{mg}, 0,4 \mathrm{mg}$, $0,2 \mathrm{mg}, 0,1 \mathrm{mg}$ in $0,1 \mathrm{ccm}$ derselben enthalten sind. Bei intrakutaner Injektion in Dosis von $0,2 \mathrm{ccm}$ jeder Lösung in den Rücken der Meerschweinchen, wurde die Hautreaktion nach 48 Stunden in verschiedener Stärke und Grösse hervorgerufen.

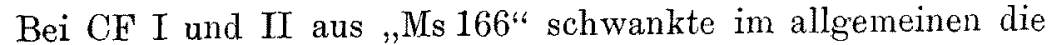
Rötung zwischen $10 \times 11 \mathrm{~mm}-4 \times 2 \mathrm{~mm}$ im Durchmesser. Nekrose war nur in einem Fall gefunden. Bei $\mathrm{PF}_{1}$ desselben Stamms fand man an allen Injektionsstellen Nekrose von $2 \times 1 \mathrm{~mm}-1 \times 1 \mathrm{~mm}$ Durch- 
messer, $16 \times 15 \mathrm{~mm}-12 \times 11 \mathrm{~mm}$ grosse Rötung, wobei die Anschwellung mit ihnen immer parallel einherging (Tab. 6 und 7). Auch bei CF I und II aus ,Ms 163" begleitete die Hautreaktion an Injektionsstellen Nekrose von $4 \times 3 \mathrm{~mm}-1 \times 2 \mathrm{~mm}$ Grösse, Rötung von $10 \times$ $14 \mathrm{~mm}-10 \times 11 \mathrm{~mm}$ und etwaige Anschwellung (Tab. 8). Zwar ist es sehr schwer, über die Hautreaktion herbeiführende Giftigkeit einzelner Fraktionen-in so wenigen Fällen-ausschlaggebendes Urteil abzugeben, denn die Reaktion ist je nach der Individualität des Tieres leicht verschiebbar, aber aus dem Befund, dass, wie man aus Tabellen erkennt, bei Stamm ,Ms 166" an Stärke die eiweissreaktionzeigenden Fraktionen den negativen weit tiberlegen sind, kann mit Wahrscheinlichkeit darauf gefolgert werden, dass bei der Hautreaktion dem Eiweiss eine Rolle zukommt.

Tabelle 6.

Intrakutane Reaktion durch CF I und II aus Stanm ,Ms $166^{\prime 4}$.

\begin{tabular}{|c|c|c|c|c|c|}
\hline \multicolumn{2}{|c|}{ Fraktion } & \multicolumn{2}{|c|}{$\mathrm{CF} I$} & \multicolumn{2}{|c|}{ CF II } \\
\hline $\begin{array}{l}\text { Injektions- } \\
\text { Dosis }\end{array}$ & 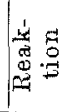 & 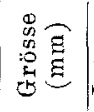 & 象语 & 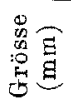 & 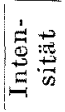 \\
\hline \multirow{3}{*}{$\begin{array}{l}1,6 \mathrm{mg} \\
(0,2 \mathrm{ccm})\end{array}$} & $N$ & $1 \times 1$ & + & & \\
\hline & $\mathbf{R}$ & $10 \times 11$ & $H$ & $7 \times 7$ & H \\
\hline & A & & + & & \\
\hline \multirow{3}{*}{$\begin{array}{l}0,8 \mathrm{mg} \\
(0,2 \mathrm{ccm})\end{array}$} & $\mathrm{N}$ & & & & \\
\hline & $\mathrm{R}$ & $5 \times 6$ & $H$ & $4 \times 4$ & + \\
\hline & A & & & & \\
\hline \multirow{3}{*}{$\begin{array}{l}0,4 \mathrm{mg} \\
(0,2 \mathrm{ccm})\end{array}$} & $\mathrm{N}$ & & & & $\cdot$ \\
\hline & $\mathrm{R}$ & $4 \times 3$ & + & $4 \times 2$ & + \\
\hline & A & & & & \\
\hline
\end{tabular}

Tabelle 7 .

Intrakutane Realtion durch PF I und II aus Stamm „Ms $166^{\prime \prime}$.

\begin{tabular}{|c|c|c|c|c|c|}
\hline \multicolumn{2}{|c|}{ Fraktion } & \multicolumn{2}{|c|}{$\mathrm{PF}_{1} \mathrm{I}$} & \multicolumn{2}{|c|}{$\mathrm{PF}_{1} \mathrm{II}$} \\
\hline $\begin{array}{l}\text { Injektions- } \\
\text { Dosis }\end{array}$ & 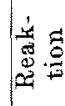 & 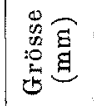 & 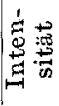 & 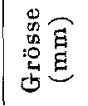 & 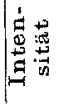 \\
\hline \multirow{3}{*}{$\begin{array}{l}0,8 \mathrm{mg} \\
(0,2 \mathrm{ccm})\end{array}$} & $\mathrm{N}$ & $2 \times 1$ & $H$ & $1 \times 1$ & + \\
\hline & $\mathbf{R}$ & $16 \times 15$ & $H$ & $17 \times 15$ & $H$ \\
\hline & A & & + & & + \\
\hline \multirow{3}{*}{$\begin{array}{l}0,4 \mathrm{mg} \\
(0,2 \mathrm{ccm})\end{array}$} & $\mathbb{N}$ & $1 \times 1$ & + & $1 \times 1$ & + \\
\hline & $\mathrm{R}$ & $13 \times 12$ & \# & $14 \times 13$ & H \\
\hline & A & & + & & + \\
\hline \multirow{3}{*}{$\begin{array}{l}0,2 \mathrm{mg} \\
(0,2 \mathrm{ccm})\end{array}$} & $N$ & $1 \times 1$ & + & $1 \times 1$ & + \\
\hline & $\mathrm{R}$ & $13 \times 11$ & H & $12 \times 11$ & 1. H \\
\hline & A & & + & & + \\
\hline
\end{tabular}

Die Grösse der Rötung und der Nekrose sind in mm ausgedrückt. $N=$ Nekrose $\mathrm{R}=$ Rötung A $=$ Anschwellung 
Tabelle 8.

Intrakutane Reaktion durch CF I und II aus Stamm "Ms $163^{\prime \prime}$.

\begin{tabular}{|c|c|c|c|c|c|}
\hline \multicolumn{2}{|c|}{ Fraktion } & \multicolumn{2}{|c|}{ CF I } & \multicolumn{2}{|c|}{ CF II } \\
\hline $\begin{array}{l}\text { Injektions. } \\
\text { Dosis }\end{array}$ & 亲 & 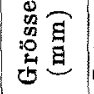 & 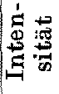 & 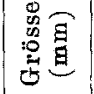 & 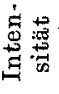 \\
\hline \multirow{3}{*}{$\begin{array}{l}1,6 \mathrm{mg} \\
(0,2 \mathrm{~cm})\end{array}$} & $\mathrm{N}$ & $4 \times 3$ & \# & $3 \times 3$ & H \\
\hline & $\mathrm{R}$ & $10 \times 14$ & H & $15 \times 15$ & \# \\
\hline & A & & $H$ & & H \\
\hline \multirow{3}{*}{$\begin{array}{l}0,8 \mathrm{mg} \\
(0,2 \mathrm{cem})\end{array}$} & N & $4 \times 2$ & \# & $2 \times 3$ & tt \\
\hline & $\mathrm{R}$ & $10 \times 8$ & H & $15 \times 12$ & H \\
\hline & A & & + & & + \\
\hline \multirow{3}{*}{$\begin{array}{l}0,4 \mathrm{mg} \\
(0,2 \mathrm{~cm})\end{array}$} & $N$ & $2 \times 3$ & $H$ & $1 \times 2$ & H \\
\hline & $\mathbf{R}$ & $10 \times 7$ & $H$ & $10 \times 11$ & $H$ \\
\hline & A & & + & & + \\
\hline \multicolumn{6}{|c|}{$\begin{array}{l}\text { Die Grösse der Rötung und der Nekrose } \\
\text { sind in mm ausgedrückt. }\end{array}$} \\
\hline $\begin{array}{l}\mathrm{N}=\mathrm{Ne} \\
\mathrm{A}=\mathrm{An}\end{array}$ & $\begin{array}{l}\text { rose } \\
\text { chwe }\end{array}$ & & & Rötung & \\
\hline
\end{tabular}

Präzipitationsversuch der Fraktionen mit Kaninchenimmunsera.

Zum Versuche dienten CF IT und $\mathrm{PF}_{1}$ II aus ,Ms 166“, und $\mathrm{CF}$ I und II aus ,Ms 163". Zur Ermittelung des Präzipitationsversuches wurden 1 Proz. Lösungen der Fraktionen in verschiedenen Verdünnungen über das rohe Kaninchenimmunserum, das mit betreffenden Mäusetyphusbazillen immunisiert ist, geschichtet. Beim positiven Ausfall trat ein weisslicher Ring an der Grenze zwischen der Lösung und dem Serum auf. Die Resultate wurden nach einer 2 stündigen Inkubation bei $37^{\circ} \mathrm{C}$ abgelesen. Wie aus Tabelle 9 ersichtlich ist, erwiesen sich diese Fraktionen, besonders CF in vitro als serologisch hoch aktiv. Vor allem präzipitierte CF

II aus ,Ms $166^{\prime \prime}$ das spezifiche Serum in einer Verdünnung von $1: 5$ $\times 10^{5)}$.

Tabelle 9 .

Präzipitation der Fraktionen mit Kaninchenimmunsera.

\begin{tabular}{|c|c|c|c|c|c|c|c|c|c|c|}
\hline \multicolumn{2}{|c|}{ Verdünnung } & \multirow{2}{*}{$\frac{10^{-2}}{4}$} & \multirow{2}{*}{$\frac{10^{-3}}{H}$} & \multirow{2}{*}{$\frac{5 \times 10^{-3}}{4}$} & \multirow{2}{*}{$\frac{10^{-4}}{4}$} & \multirow{2}{*}{$\frac{2 \times 10^{-1}}{H}$} & \multirow{2}{*}{$\frac{5 \times 10^{-4}}{H}$} & \multirow{2}{*}{$\frac{10^{-5}}{H}$} & \multirow{2}{*}{$\frac{2 \times 10^{-5}}{4}$} & \multirow{2}{*}{$\frac{5 \times 10^{-5}}{+}$} \\
\hline \multirow{4}{*}{ 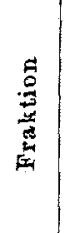 } & $\stackrel{C}{0}: \mathrm{CF}$ II & & & & & & & & & \\
\hline & $\sum_{=}^{\infty} \mathrm{PF}_{\mathrm{I}} \mathrm{II}$ & Ht & $H$ & m & + & + & & & & \\
\hline & CF I & H & H & H & $H$ & $H$ & + & & & \\
\hline & $\sum_{=}^{\infty}|\mathrm{CF} \mathrm{II}|$ & $H$ & $H$ & $H$ & $H$ & + & & & & \\
\hline
\end{tabular}

Uber die Ausbeutederextrahierten Substanzen

aus beiden Stämmen und überdieselbe

derdaraus isolierten Fraktionen.

Im Vergleich der Ausbeute an die extrahierten Substanzen aus 
beiden Stämmen und an die daraus isolierten Fraktionen kam man zum folgenden Ergebnis.

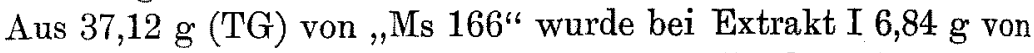
Trockensubstanz ausgezogen, also 18,4 Prozent. Trockensubstanz der Bakterien schon durch Waschen und Umrührung ins Wasser übergegangen, hingegen bei Extrakt II 9,0 g trockene Substanz, also 34 Proz. der Residuemenge (26,45 g). Bei „Ms 163 “ entspricht die ins Extrakt I extrahierte Substanz $(11 \mathrm{~g})$ auf das Trockenbakteriengewicht $(44,096 \mathrm{~g})$ bezogen 24,94 Proz., dagegen die ins Extrakt II ausgezogene 16,746 g, gleicherweise gerechnet wie oben, 53,98 Proz. Die Gesamtmenge der

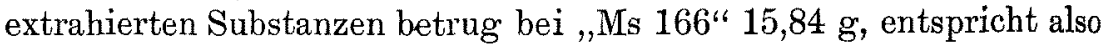
42,7 Proz. Trockenbakteriengewicht, hingegen bei ,Ms 163 " $27,746 \mathrm{~g}$, also 62,92 Proz. Aus diesen Ziffern lässt sich entnehmen, dass stark virulente Mäusetyphusbazillen als schwach virulente viel leichterzerstörbar sind und viel leichterihre Leibessubstanzen in ihr Medium abgeben können. Was die Ausbeute an isolierte Fraktionen betrifft, so gelangte man zu folgendem sehr interessanten Resultat. Die Gesamtmenge der aus beiden Extrakten von „Ms 166" erhaltenen PF belief sich auf 5,9722 g, machte also 37,7 Proz. der gesamten extrahierten Substanzen aus, von denen die PF II 85, 9 Proz., hingegen PF I nur 14,1 Proz. in Anspruch nahm. Das Gewicht von CF I und II betrug im ganzen $0,4585 \mathrm{~g}$, entspricht also 2,88 Proz. der gesamten extrahierten Substanzen. Von $0,4585 \mathrm{~g}$ fällt auf CF I 0,3625 g und auf CF II 0,096 g. Daraus lässt sich verstehen, dass schon durch das erste Verfahren 79,1 Proz. der ganzen CF extrahiert war.

Die obenangegebenen Ergebnisse berechtigen zur Annahme, dass bei schwach virulenten Mäusetyphusbazillen, während CF vorwiegend die Oberfläche des Bakterienleibes bildet und leicht zu extrahieren ist, PF den Hauptbestandteil desselben darstellt und erst durch Zerreiben frei gemacht werden kann.

Wenn man sich nun zum „Ms 163 “ wendet, so sieht man, dass bei diesem Stamm PF nur in unbeträchtlich kleiner Menge $(0,0234 \mathrm{~g})$ ausschliesslich aus Extrakt II mittels Trichloressigsäure $(\mathrm{pH} 1,2)$ isoliert war, während CF I und II in allem 12,537 $\mathrm{g}$ betrug.

Von CF I und II macht auf das ganze Gewicht gerechnet, die erstere 7,37 Proz. und die letztere 92,6 Proz. aus. Aus diesem Ergebnisse geht hervor, dass sehr stark virulente Mäusetyphusbazillen von schwach virulenten strukturell deutlich verschieden sind, $d$. h. die Leiber der stark virulenten Bakterien meistenteils aus sog. Kohlenhydrat- 


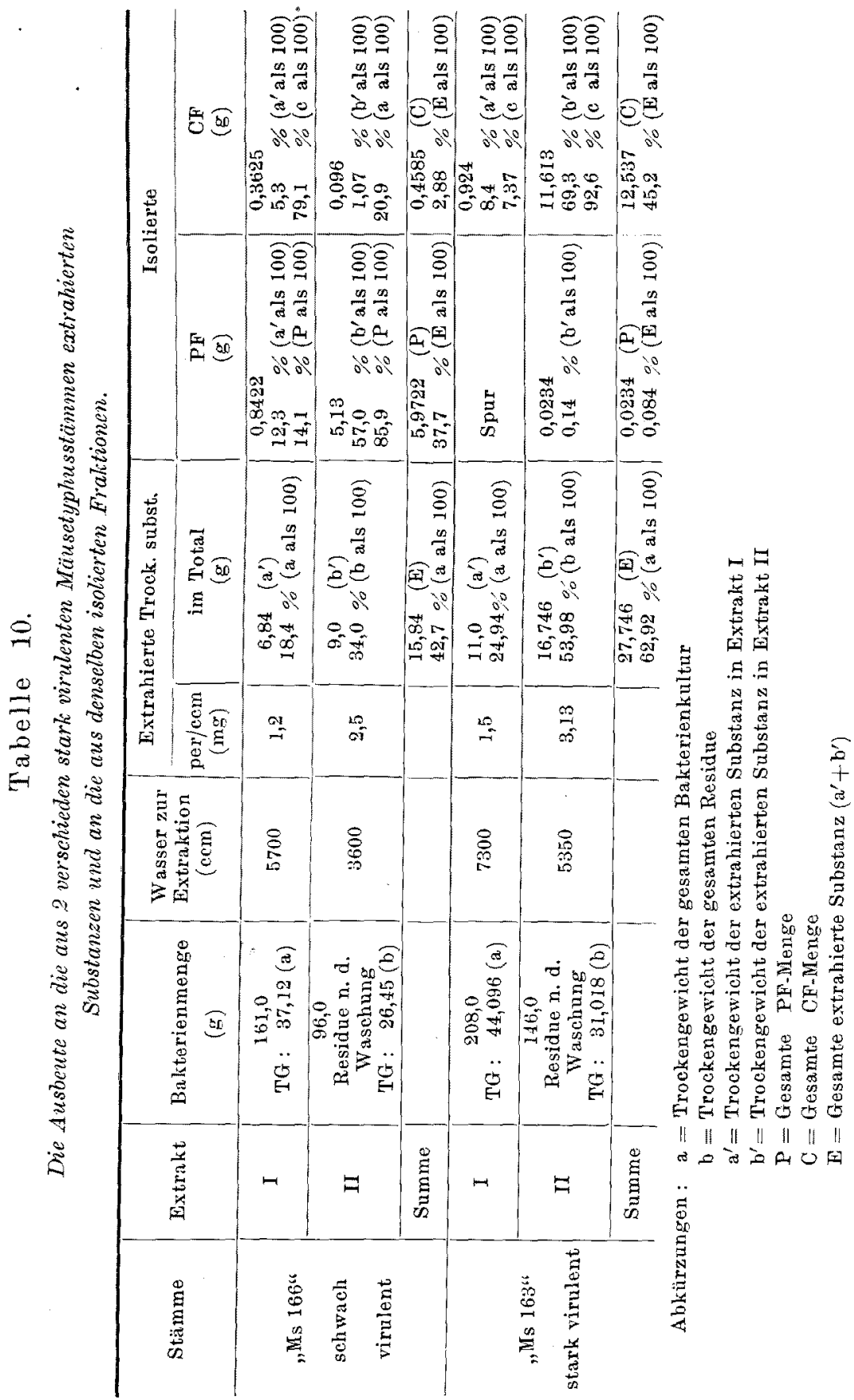


Komplex, welcher löslich in schwach saurer Reaktion und zugleich sehr toxisch für Mäuse ist, gebaut sind (Tab. 10).

Die chemischen Eigenschaften der Fraktionen.

Zu qualitativen chemischen Reaktionen wurde eine 1 Proz. Lösung jeder Fraktion herangezogen (Tabelle 11).

Farbenreaktion.

Die Farbenreaktion für Eiweiss fiel bei PF ganz entschieden positiv und bei CF etwas schwächer positiv aus. CF I und II aus beiden Stämmen zeigten Biuretreaktion, Pauly sche Diazoreaktion und Sakagutische Reaktion. Die Molischsche war vor allem bei beider CF I am stärksten und bei CF II aus ,Ms 163" mittelmässig stark. Ninhydrinprobe konnte an CF I und II aus "Ms 166" aus Mangel an Material nicht durchgeführt werden.

Koagulationsversuch.

PF-Lösung koagulierte durch Erhitzung in schwach saurer Reaktion, dagegen nicht in neutraler Reaktion, während CF I und II in beiden Reaktionen nicht koagulierten. Hellersche Probe war nur bei $\mathrm{PF}$ positiv. Wie schon erwähnt, waren CF I und II aus beiden Stämmen mit Alkohol ausfällbar.

\section{Präzipitationsversuch.}

PF-Lösung wurde durch alle Alkaloidreagenzien präzipitiert. Natriumsulfat, Ammoniumsulfat und Phosphorwolframsäure erzeugten in CF II aus "Ms 163" einen starken flockigen Niederschlag, doch die üblichen Reagenzien nur Trübung.

Kohlenhydratreation.

CF I und II aus beiden Stämmen reduzierten Fehlingsche Lösung spärlich in statu, im Gegensatz hierzu nach einer 5stündigen Hydrolyse mit 3 Proz. Salzsäure sehr deutlich, PF-Lösungen reduzierten es spurweise, wenngleich nach einer mehrstindigen Hydrolyse.

Für Orcin-Salzsäure-Reaktion (Pentose oder Glukuronsäure) und Phloroglucin-Salzsäure-Reaktion (Pentose oder Glukuronsäure) ergaben alle Lösungen ein positives Resultat. Als ein Stück vorher in. Anilinazetat eingetauchtes Filterpapier dem Dampf, welcher erst nach der Abdampfung der mit Salzsäure erhitztenen zu prüfenden Lösungmit A usnahme von PF_-entsteht, ausgesetzt wurde, verfärbte es sich rosarötlich. Das spricht auch für die Anwesenheit einer Zuckerkom- 
Tabelle 11.

Die chemischen Eigenschaften der verschiedenen Fraktionen.

\begin{tabular}{|c|c|c|c|c|c|}
\hline \multirow{2}{*}{\multicolumn{2}{|c|}{$\underbrace{\text { Extrakt }}_{\text {Stamm }}$}} & \multicolumn{2}{|c|}{ "Ms 166" } & \multicolumn{2}{|c|}{ "Ms $163^{46}$} \\
\hline & & I, II & I, II & $\mathbf{I}$ & II \\
\hline & Fraktion & CF & PF & $\mathrm{CF}$ & $\mathrm{CF}$ \\
\hline \multirow{11}{*}{ 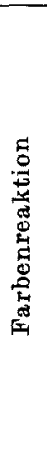 } & Binretreaktion & + & + & + & + \\
\hline & Xanthoproteinreaktion & \pm & + & \pm & + \\
\hline & Millo n sche Reaktion & \pm & + & + & + \\
\hline & Hopkins-Colesche Reaktion & - & + & + & + \\
\hline & Neubauer-Rhod esche Reaktion & - & + & - & \pm \\
\hline & Ninhydrinreaktion & & + & + & + \\
\hline & PbS-Reaktion & - & \pm & - & - \\
\hline & Molis ch sche Reaktion & H & - & HH & H \\
\hline & Paulysche Diazoreaktion & \pm & + & + & + \\
\hline & Sakagutische Reaktion & + & + & + & + \\
\hline & Liebermannsche Reaktion & $=$ & \pm & $=$ & - \\
\hline
\end{tabular}

\begin{tabular}{|c|c|c|c|c|c|}
\hline \multirow{3}{*}{ 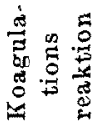 } & Erhitzung in neutraler Reaktion & - & - & - & - \\
\hline & Erhitzung in saurer Reaktion & - & + & - & - \\
\hline & Hellersche Reaktion & - & + & - & - \\
\hline
\end{tabular}

\begin{tabular}{|c|c|c|c|c|c|}
\hline \multirow{9}{*}{ 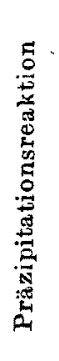 } & Trichloressigsänre & \pm & $H$ & + & + \\
\hline & Sulfosal cylsäure & - & H & + & + \\
\hline & $\mathrm{Na}_{2} \mathrm{SO}_{4}$ & \pm & H & + & $H$ \\
\hline & 2/3 Sittigung mit $\left(\mathrm{NH}_{4}\right)_{2} \mathrm{SO}_{4}$ & + & Ht & $H$ & int \\
\hline & $25 \%$ Quecksilberazetat & \pm & $H$ & + & + \\
\hline & Phosphorwolframsäure & + & $\mathrm{H}$ & + & $H$ \\
\hline & Bleisubazetat & + & H+ & + & + \\
\hline & Tanninsäure & + & $H$ & \pm & + \\
\hline & $\mathrm{NaCl}$ & - & + & + & + \\
\hline
\end{tabular}

\begin{tabular}{|c|c|c|c|c|c|}
\hline \multirow{5}{*}{ 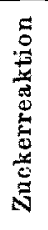 } & $\begin{array}{l}\text { Reduktion der FehIing schen Lös. } \\
\text { nach Hydrolyse mit } 3 \% \text { HCl }\end{array}$ & + & \pm & + & + \\
\hline & Orcin-HCl-Reaktion & + & + & + & \pm \\
\hline & Phloroglucin-HCl-Reaktion & & + & $t$ & + \\
\hline & Anilinazetatreaktion & + & \pm & + & + \\
\hline & Glukosaminreaktion & + & \pm & + & + \\
\hline
\end{tabular}

\pm spur

+ schwach positiv, Trübung (bei Präz.versuch)

H stark positiv, Flockung (bei Präz.versuch) 
Tabelle 12.

$N, P-$, Asche- und Zuckergehalt von verschiedenen Fraktionen.

\begin{tabular}{|c|c|c|c|c|c|c|}
\hline \multirow{2}{*}{\multicolumn{3}{|c|}{$\frac{\text { Stamm }}{\text { Extrakt }}$}} & \multicolumn{2}{|c|}{ "Ms $166^{4}$} & \multicolumn{2}{|c|}{ "Ms $163^{4}$} \\
\hline & & & \multicolumn{2}{|c|}{$I$} & I & II \\
\hline \multicolumn{3}{|l|}{ Analyse } & $\mathrm{CF}$ & $\mathrm{PF}$ & $\mathrm{CF}$ & $\mathrm{CE}$ \\
\hline \multicolumn{3}{|c|}{$N$} & $2,27 \% 6$ & $13,2 \%$ & $2,19 \%$ & $6,69 \%$ \\
\hline \multicolumn{3}{|c|}{$p$} & $3,6 \%$ & $0,84 \%$ & $0,45 \%$ & $0,59 \%$ \\
\hline \multirow{7}{*}{$\begin{array}{l}\text { Reduzierende } \\
\text { Substanz }\end{array}$} & \multirow{7}{*}{ 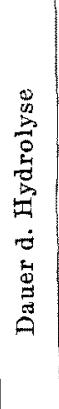 } & 0 & $3,0 \%$ & 0 & $1,8 \%$ & 0 \\
\hline & & $1 \mathrm{St}$. & 30 & 0 & $55,1 \%$ & $6,75 \%$ \\
\hline & & $11 / 2 \mathrm{St}$. & $32,6 \%$ & $1 \%$ & $59,9 \%$ & $17,94 \%$ \\
\hline & & $2 \mathrm{st}$ & $32,8 \%$ & $2 \%$ & $52,67 \%$ & $10,48 \%$ \\
\hline & & 3 St. & 32,2 \%о & $8,2 \%$ & $51,74 \%$ & $8,39 \%$ \\
\hline & & $4 \mathrm{St}$. & 30,8 \% & $9 \%$ & $51,09 \%$ & $7,22 \%$ \\
\hline & & $6 \mathrm{st}$. & $17,6 \%$ & $7,8 \%$ & 18,57 gó & $0,26 \%$ \\
\hline \multicolumn{3}{|c|}{ Asche } & $12,5 \%$ & $2,51 \%$ & 12,5 ó & $16,6 \%$ \\
\hline
\end{tabular}

ponente (Pentose oder Glukuronsäure). Unter allen Fraktionen zeigten insbesondere CF nach Alkalisierung mit dem Ehrlichschen Reagens eine für Glukosamin charakteristische Reaktion.

Stickstoff, Phosphor, Reduktionskraft und Asche wurden auch quantitativ bestimmt ( $N$ nach Mikrokjeldahl, Phosphor nach Lohmann-Jendrassik und Reduktionskraft nach HagedornJensen) (Tab. 12). Die Resultate sind in Tabelle 12 zusammengefasst. Die Beurteilung jeder Fraktion lautet aus obigen Eigenschaften folgenderweise, aber zuvor sei hier vorteilshalber über CF II aus „Ms 163 " besprochen.

Bei ihr

1. fällt die Farbenreaktion für Eiweiss und Kohlenhydrat positiv aus,

2. tritt durch Erhitzung in neutraler und saurer Reaktion keine Koagulation auf,

3. wird nach Zusatz von 2 Raumteilen gesättigter Ammoniumsulfatlösung ein auffallender flockiger Niederschlag erzeugt,

4. ist es mit Alkohol ausfällbar,

5. sind ferner Phloroglucin-Salzsäure, Orcin- und Glukosaminreaktionen positiv. 
Aus diesen Eigenschaften geht mit aller Wahrscheinlichkeit hervor, dass CF II aus ,Ms 163“ eine glukoproteinartige Verbindung, bestehend aus Eiweiss und Kohlenhydrat, oder ein Gemisch derselben darstellt, worüber in fortsetzender II. Mitteilung noch genauer gesprochen werden wird.

Auf Grund aller chemischen Eigenschaften wurde wohl auch der Beweis erbracht, dass eine Art Eiweiss in PF aus "Ms 166" und eine Art Polysaccharid in CF I aus ,Ms 166" und „Ms 163“ enthalten sind. Da aber einerseits bei PF die Kohlenhydratreaktion und andererseits bei CF I die Eiweissreaktion leicht bewiesen sind, so liegt die Vermutung nahe, dass diese Substanzen nicht einmal einheitlich, sondern mit anderer Komponente gemischt sein, wovon noch im folgenden die Rede sein wird. CF I aus „Ms 163" scheint nach Gehalt an reduzierender Substanz (59,9 Proz.) und an geringem Stickstoff dem sog. reinen Polysaccharid sehr nahe verwandt zu sein.

Wegen zu geringer Ausbeute konnte der Versuch an PF aus „Ms $163^{\prime \prime}$ nicht angestellt werden, jedoch kann man diese Fraktion mit der

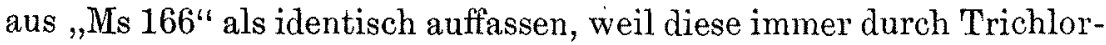
essigsäure ( $\mathrm{pH} 1,2)$ ausgefällt wurde.

\section{Besprechung.}

Aus der schon im Jabre 1933 von Tomscik und Szongott $t^{31)}$ gemachten Äusserung, dass bei der Virulenz der Milzbrandbazillen dem Polysaccharid überhaupt keine Rolle zugeschrieben wird, und ferner aus der von I ván ovi $\mathrm{cs}^{32)}$ neulich bekannt gemachten Mitteilung, dass sich Anthrax-Polysaccharid aus der virulenten und a virulenten Varietät desselben Stamms in gleichen Mengen herausnehmen liess, ist leicht zu entnehmen, dass bei diesen Bakterien das Polysaccharid sowohl quantitativ wie auch qualitativ mit der Virulenz nichts zu tun hat. In den Versuchen über die Beziehung zwischen der Virulenz und der Antigenstruktur von Salmonellen (S. aertrycke) gewann Bo i vi i ${ }^{33)}$ mittels Trichloressigsäure aus zwei S-Varianten den sog. antigène glucidolipidique 0 von gleicher Menge und von chemisch und biologisch fast gleichen Eigenschaften, ungeachtet ihrer Verschiedenheit in Virulenz. Jedoch aus dem R-Variant konnte er ihn gar nicht gewinnen. Nach Pike und Mackenzie ${ }^{34)}$, die sich auch mit diesem Gegenstand mit schwach und stark virulenten glattförmigen Stämmen der Mäusetyphusbazillen beschäftigten, besteht $\mathrm{z}$ wischen den Polysacchariden aus beiden Stämmen chemisch und toxisch keine merkliche Differenz, so dass sie für die Virulenz nicht entscheidend sind. Der Hauptfaktor, 
der die Virulenzverschiedenheit herbeiführt, ist nach ihnen die Fähigkeit, mit der sich die stark virulenten Bakterien im Tierkörper viel lebhafter vermehren als die schwach virulenten.

Kuroya und seine Mitarbeiter ${ }^{30)}$ fanden in ihrem Versuche an Typhusbazillen, dass die Leibessubstanz von stark virulenten Bakterien viel leichter als die von schwach virulenten in Lösung überging, und schlossen ferner daraus, dass dieser Unterschied eine der Ursachen der Virulenzverschiedenheit sein kann, vorausgesetzt, dass ein solcher auch in vivo eventuell möglich sei. Diese Forscher, und später Ku roya und Nisimura ${ }^{25)}$ konnten ferner feststellen, dass die M.L.D. der PF und CF bei Typhusbazillen 2,6 $\mathrm{mg}$ und $0,4 \mathrm{mg}$, und die der PF bei Cholerabazillen 2,5 mg betrug, also vom ganzen gesehen, CF giftiger als PF, doch quantitativ die letztere der ersteren weit überlegen ist, und dass sogar aus stark virulenten Bakterien mehr CF als aus schwach virulenten herausgenommen wird. Sie kamen also durch diesen Versuch zu dem Resultat, dass die Virulenz eines Stamms sowohl auf PF als auch auf $\mathrm{CF}$ zurückgeführt werden soll.

An Hand des eigenen Versuches konnte gezeigt werden, dass schwach virulente Mäusetyphusbazillen sowohl an Ausbeute der Extrakte und der Fraktionen als auch in ihrer Toxität den schwach virulenten Typhusbazillen gleichzusetzen waren, während bei stark virulenten Mäusetyphusbazillen PF einen unbeträchtlich kleinen Teil, hingegen CF den grössten Teil ausmacht. Ferner, da CF II 92,6 Proz. der gesamten CF-Menge entspricht, so geht es nicht zu weit zu sagen, dass bei diesen Bakterien CF II den Hauptbestandteil bildet. Von CF II wird in der nächsten Mitteilung eingehend die Rede sein.

\section{Schlussfolgerungen.}

1. Es wurde an Mïusetyphusbazillen ein Versuch gemacht, in welchem nach einer Beziehung zwischen der Virulenz und den giftigen Substanzen gesucht wurde.

2. Die Leibessubstanzen sind bei stark virulenten Bakterien leichter zu extrahieren als bei schwach virulenten.

3. Schwach virulente Bakterien bestehen aus Eiweiss, das den Hauptbestandteil der Körper bildet, und wenig Polysaccharid. Stark virulente Bakterien setzen sich dagegen vorwiegend aus einem Kohlenhydrat-Eiweisskomplex zusammen, dessen Einheitlichkeit noch kaum bestätigt ist.

4. Unter allen Fraktionen, die sich sämtlich für Mäuse giftig verhalten, ist $\mathrm{CF}$ am stärksten. 
5. Die Virulenz kommt infolgedessen wenigstens zu einem Teile dem Gehalt an bakterienleibbildender CF zu.

Diese Arbeit wurde von dem Saito-Hoonkai unterstützt, wofür Verfasser ihm herzlichsten Dank ausspreche.

\section{Literatur.}

(1) Kruse, W., Microbiol., Leipzig 1910, 1021.

(2) Wyssokowitsch, W., Zeitschr. f. Hyg, 1886, 1, B.

(3) G a ma leia, N., Zit. aus Hdb. d. path. Mikrorg., 1929, 1, 456.

(4) Kruseu. Bon a duce, Tbid,, 456.

(5) B ail, O., Zentralbl. f. Bakt., 1900, 27, 517.

(6) Gál, F., Zeitschr. f. Immunf., 1912, 14, 685.

(7) Thiele, F.H., u. Embelto n, D., Arch. d. med. exp. et d'anat. path., 1914, 26, 60 ; Zeitschr. f. Immunf., 1913, 19, 643 .

(8) Jensen, Zit. ans Hdb. d. path. Mikrorg., 1929, 1, 464.

(9) Wassermann, A., n. Ostertag, R., Monatsh. f. prak, Tierheilkunde., 1902, 13,466 .

(10) Jaffé, J., u. Besserer, A., Deutsch. med. Wochensch., 1905, 2044.

(11) Preis z, H., Zentralbl. f. Bakt. Y. Orig., 1907, 44, 209 ; ebenda, 1907, 47, 585.

(12) Gruber, M., u. Futaki, K., M. m. W, 1907, 54, 249.

(13) Ba il, 0., Zentralbl. f. Bakt. I. Orig., 1915, 75, 159.

(14) Stam atin, N., Archiva veterinara, 1934, $26,1$.

(15) Sohater, W., C. r. Soc. Biol., 1936, 122, 897.

(16) Sterne, M., Zit. aus Zentralbl. f. Bakt. I. Orig., 1940, 146, 18.

(17) von B z za, L., Zentralbl. f. Bakt. I. Orig., 1939, 143, 244 ; ebenda 1940, 146, 18.

(18) Voges, O., Zentralbl. f. Bakt. I. Orig., 1896, 20, 906.

(19) Koske, F., Arb. a. d. K. Gesundheitsamte, 1906, 24, 305.

(20) Branham, S. E., J. Infect. Dis., 1925, 37, 291.

(21) Eoker, E. E., J. Infect. Dis., 1917, 21, 541.

(22) Ecker, E. E., u. Riming to n, C., J. Hyg., 1928, 27, 44.

(23) Casper, W., Zeitschr. f. Hyg., 1928, 109, 170.

(24) Menten, M. L., u. King, C. G., J. Infeet. Dis., 1930, 46, 275.

(25) Furth, J., u. Land steiner, K., J. of Exp. Med., 1929, 49, 11.

(26) Raistrick, H., u. Topley, W. W. G., Brit. J. Exp. Path., 1934, 15, 113.

(27) Boivin, A., u. Mesrobean u, K., Revue d'Immun., 1935, 1, 555.

(28) I ván o vic s, G., Zeitschr. f. Immunf., 1936, 87, 310.

(29) Hos y a, S., u. Ka washima, S., Zikken Igaku, 1936, $20,913$.

(30) Kuroya, M., Osio, K., u. Pin. K. H. Tohoku J. Exp. Med., 1940, 38, 167.

(31) Tom seik, J. u. S zong ot t, H., Zeitschr. f. Immunt., 1932, 76, 214.

(32) I vánovies, G., Zeitschr. f. Immunf., 1940, 97, 402.

(33) Boivin, A., C. r. Soc. Biol., 1939, 132, 370.

(34) Pike, R. M., u. Mackenzie, G. M., J. Bact., 1940, 40, 171 u. 197.

(35) K u r o y a, M., u. N is i m u ra, H., J. Shanghai Science Inst., 1939, 4, 159. 